\title{
VIBRATIONS REJECTION IN GYROSCOPES BASED ON PIEZORESISTIVE NANOGAUGES
}

\author{
Federico Giacci, Stefano Dellea, Antonio F. Longoni, and Giacomo Langfelder \\ Dipartimento di Elettronica, Informazione e Bioingegneria, Politecnico di Milano, Italy
}

\begin{abstract}
The work discusses the effects of vibrations on the performance of rate gyroscopes in terms of Allan variance, and presents the results of vibrations rejection on a Z-axis gyroscope based on piezoresistive nano-gauge sensing elements. In a comparative analysis with a consumer offthe-shelf gyroscope, the proposed device shows a 10-fold better angle random walk (ARW) under no vibrations, and at least a 100-fold better Allan variance, when acquired under vibration amplitude of $\pm 6 \mathrm{~g}$ (gravity units), at frequencies up to $10 \mathrm{kHz}$.
\end{abstract}

\section{KEYWORDS}

MEMS gyroscopes, NEMS, piezoresistive sensing, Allan variance, vibrations rejection

\section{INTRODUCTION}

The increasing use of microelectromechanical system (MEMS) gyroscopes in several areas, such as automotive, consumer and military fields, leads to focus the attention on the repeatability of the performance of these devices in presence of shocks and/or vibrations that represent realistic environmental conditions, where they will be operated. Vibrations can occur up to few $\mathrm{kHz}$ in consumer applications, and up to $10-50 \mathrm{kHz}$ in automotive, industrial, aerospace or military environment [1].

As the operating resonance frequency of MEMS gyroscopes is commonly in the order of a few tens $\mathrm{kHz}$, it is necessary to investigate the alteration of the device behavior in presence of external vibrations. These considerations imply two main challenges: the first one is the design of gyroscope structures with maximized immunity to vibrations and shocks $[2,3]$; the second one is the quantification of the vibration effects on the device performance, typically in terms of achievable white noise and long-term stability [4].

In this paper we present a structure of a piezoresistive MEMS gyroscope [5], highlighting the main structural choices that allow good vibration rejection (Section II). All the resonant modes up to $50 \mathrm{kHz}$ are discussed. In Section III the driving and sensing discrete-component electronics used for these devices is presented, together with the overall experimental setup. In Section IV we present the experimental results of a comparison between the proposed piezoresistive gyroscope and an off-the-shelf capacitive gyroscope. The devices are subjected to vibration amplitudes of $\pm 6 \mathrm{~g}$ (gravity units), at a sweeping frequency up to $10 \mathrm{kHz}$, while their output is acquired and analyzed, both in steady conditions and under angular rates. A consistent worsening and no worsening in angle random walk performance are observed on the capacitive and piezoresistive device respectively.

\section{DEVICE DESCRIPTION}

The device under analysis is a $\mathrm{Z}$-axis gyroscope based on piezoresistive nano-gauge readout [5], a technology and a principle first introduced in [6]. The gyroscope is operated in conventional amplitude-modulation (AM), Coriolis rate, mode-split configuration [7-9]. It features drive-mode comb fingers, NEMS gauges for piezoresistive sensing, and electrodes for tuning and quadrature compensation purposes. Fig. 1 is a scanning electron microscope (SEM) picture of the device: the in-plane, anti-phase, drive mode, featuring tuning fork springs, is designed to be at about 20 $\mathrm{kHz}$. The rate signal, induced by the Coriolis (decoupling) frame on the levered sense mode, is detected through differential resistive readout: the gauge elements, positioned as shown, have a nominal cross-section of $(250 \mathrm{~nm})^{2}$ and a nominal length of $5 \mu \mathrm{m}$.

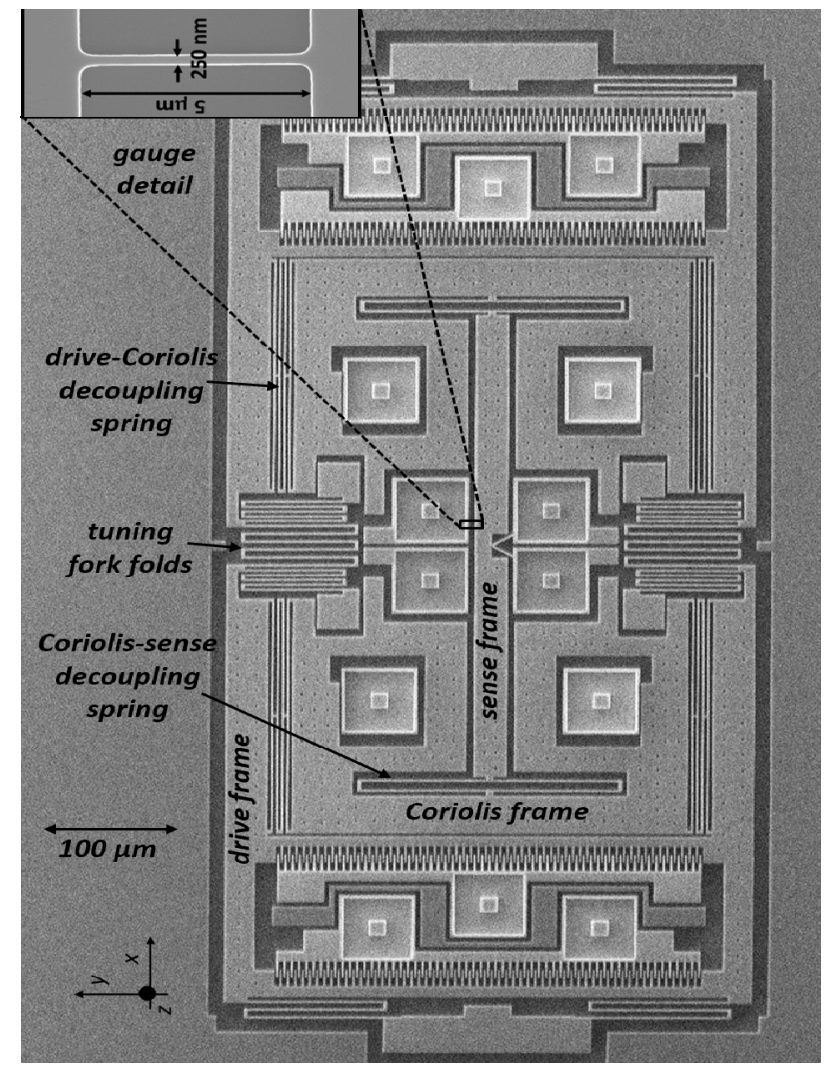

Fig. 1: SEM view of the 15- $\mu m$-thick, Z-axis gyroscope with piezoresistive readout. The tuning fork and springs for double decoupling are shown. The inset shows a gauge detail. Note the lever system positioned at the device center. 
As shown in the figure, a doubly-decoupled geometry with anchored drive frame was adopted, in order to improve immunity to vibrations as suggested e.g. in [2]. This full decoupling minimizes the transmission of movements induced by vibrations on the drive frames to the sense direction. The structure features a central, single-lever, sense frame, anchored through a rotational hinge: such a solution is particularly recommended in order to efficiently reject large common-mode inertial forces (e.g. large shocks along $\mathrm{x}$ and $\mathrm{y}$ axis, and corresponding vibrations).

Though the structure was conceived for consumer applications, the modes of the structure up to $50 \mathrm{kHz}$ (so, within the expected range of mechanical vibrations in automotive and military applications) were studied and simulated. Fig. 2 shows the results of Comsol Multiphysics finite element method (FEM) simulations for all the modes within the mentioned frequency range. For every pair of sub-figures, part A shows the full device view, and part B is a detail on the gauges region. Dark areas undergo minimum displacements, while light gray areas correspond to elements with maximum displacement.
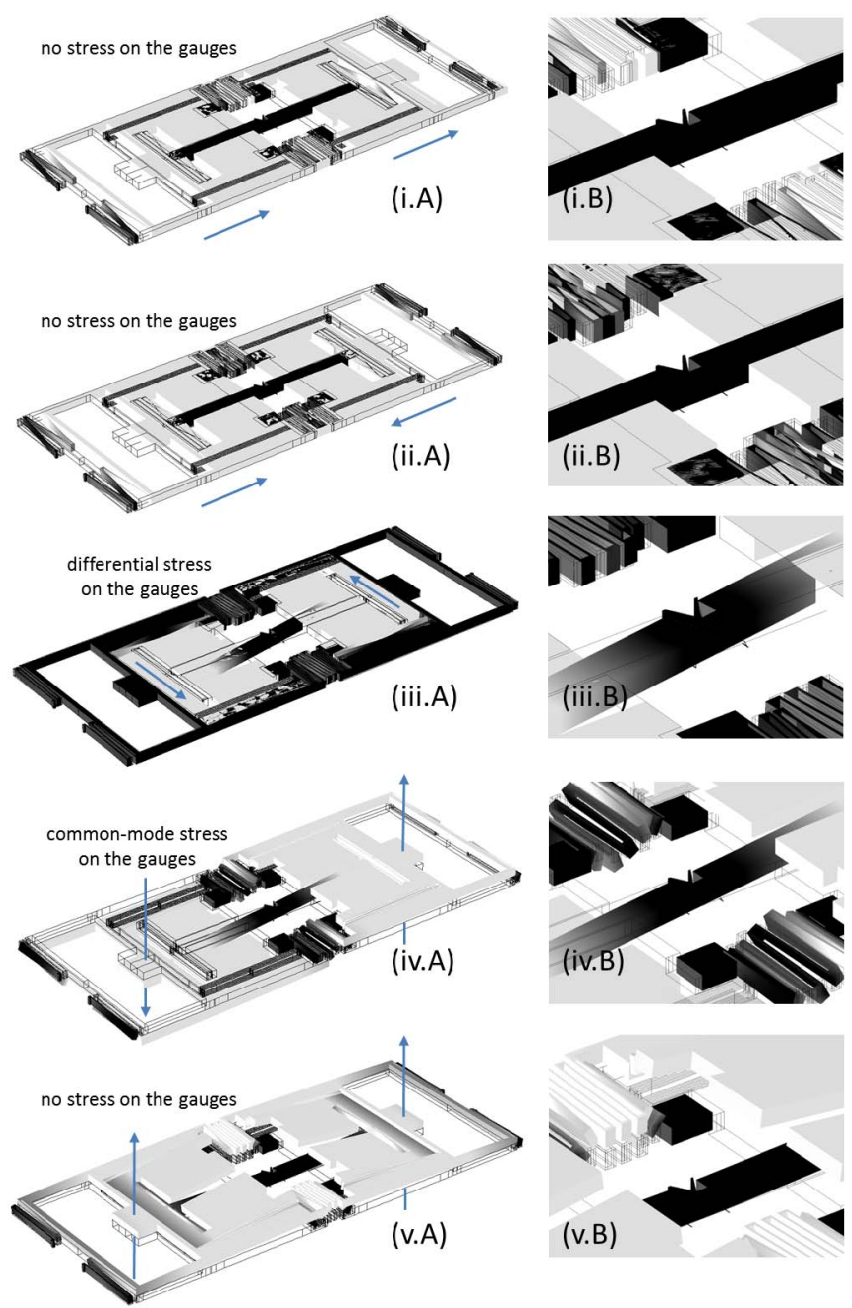

Fig. 2: the five resonant modes of the Z-axis MEMS gyroscope occurring within the $50 \mathrm{kHz}$ range. Subfigures A show the full device; $B$ are details of the hinge-gauge area.
The first mode (at about $14 \mathrm{kHz}$ ) is the in-phase drive mode. The second one (at about $19.5 \mathrm{kHz}$ ) is the tuningfork, anti-phase drive mode. For both modes, no appreciable stress on the NEMS gauges is observed thanks to the double decoupling.

The third mode (at about $20 \mathrm{kHz}$ ) represents the antiphase resonant frequency of the sense frames, under which the NEMS gauges correctly undergo a differential stress. This is indeed the excited motion when an angular velocity occurs.

The fourth $(\sim 43.1 \mathrm{kHz})$ and fifth $(\sim 46 \mathrm{kHz})$ modes represent coupled and uncoupled out-of-plane displacement of the sense frames. The former corresponds to a common mode stress for the NEMS gauges with a very small axial component. The latter ideally determines negligible stress on the gauges.

The system is thus designed to be well insensitive to shocks and vibrational stimuli within the $50 \mathrm{kHz}$ range.

\section{ELECTRONICS AND SETUP}

In order to operate the MEMS gyroscope, a discretecomponent printed circuit board (PCB) was developed, whose block scheme is represented in Fig. 3 [5]. The board includes both the drive oscillator and the sense interface, and it is compatible with mounting on both the available vibrational shaker and rate table.

The primary loop, forming the oscillator, creates the self-sustained motion of the driven masses. A secondary loop acquires the front-end output (proportional to the velocity of the driven masses) integrates it to get a signal proportional to the displacement (the quantity to be controlled), extracts its amplitude through a rectifier, compares it with a reference and generates an error signal that will control the variable gain amplifier (VGA).

The nano-gauges differential stress, caused by angular rates, is readout using a Wheatstone bridge configuration, followed by an instrumentation amplifier (INA) and a laboratory lock-in amplifier. Its output is acquired and digitized through an external analog-to-digital converter. The off-the-shelf capacitive gyroscope used for comparisons has itself an analog output that is acquired in the same way.

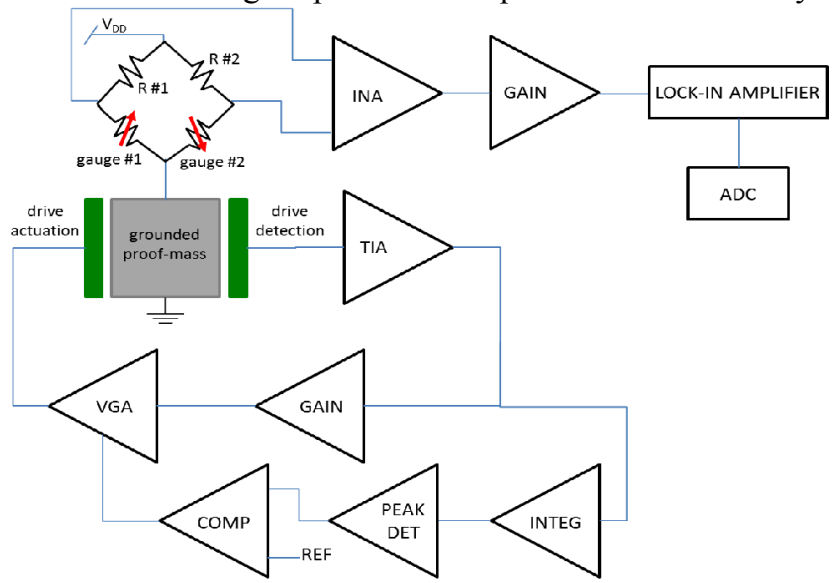

Fig. 3: block diagram representing drive and sense electronics for the device. 


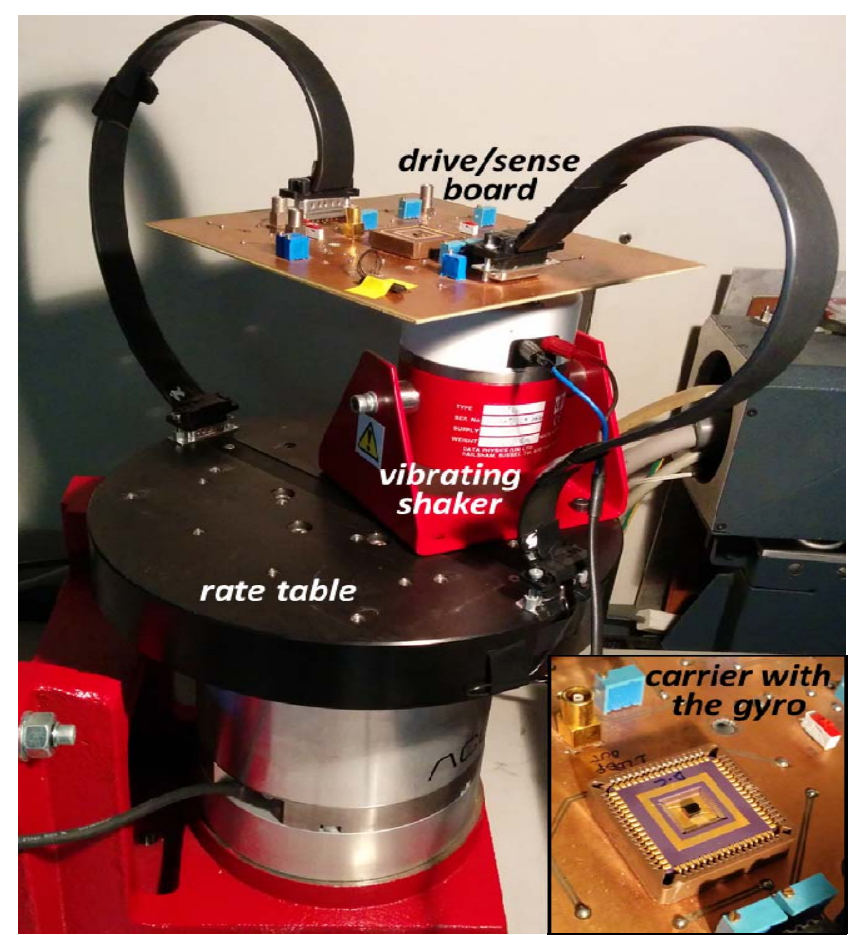

Fig. 4: the experimental setup includes a rate table, a vibrating shaker on top of it, and a board with drive and sense electronics. The inset shows the MEMS wire-bonded to a ceramic carrier, positioned inside the plastic package.

As shown in Fig. 4, the board is mounted on top of a vibrating mini-shaker, which is itself mounted on top of a rate table. This sophisticated setup simultaneously allows the application of controlled rotations and vibrations.

\section{EXPERIMENTAL RESULTS}

The off-the-shelf gyroscope has a measured sensitivity of $0.67 \mathrm{mV} / \mathrm{dps}$. The sensitivity of the piezoresistive gyroscope, using a drive-mode amplitude nominally controlled to $4 \mu \mathrm{m}$, is set to $1.87 \mathrm{mV} / \mathrm{dps}$, comparable to the capacitive system. Fig. 5 shows the measured output for both the devices under rates in the range of $\pm 1500 \mathrm{dps}$.

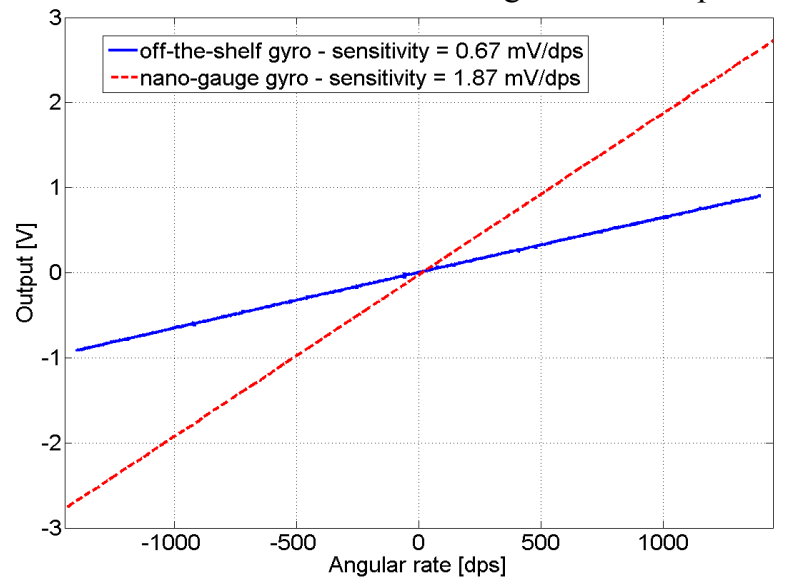

Fig. 5: measured sensitivities of the piezoresistive and the off-the-shelf consumer capacitive gyroscopes. The test was limited to $\pm 1500 \mathrm{dps}$ (the full-scale of the latter).
The devices are then subject to vibration amplitudes of $\pm 59 \mathrm{~m} / \mathrm{s}^{2}$, at a sweeping frequency up to $10 \mathrm{kHz}$. Fig. 6 shows the time-dependent output signals of the two gyroscopes, acquired during the variation (linear in time) of the injected vibration frequency. The output signal is captured without any additional rate applied to the devices.
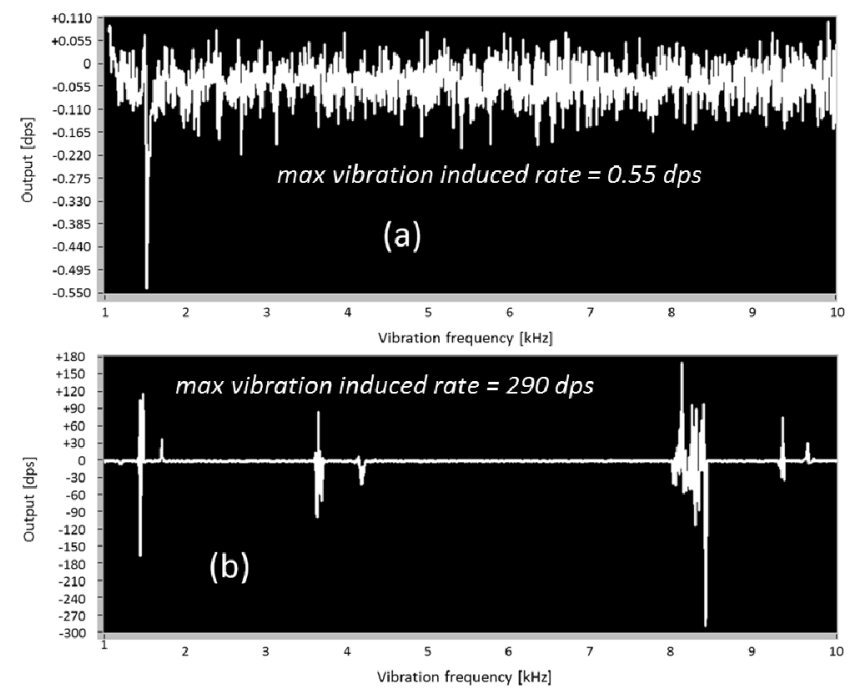

Fig. 6: gyroscope output acquired during a frequency sweep for the piezoresistive (a) and for the reference gyroscope (b). Note the different $y$-axis scales. The large vibration rejection of the proposed device is demonstrated.

Fig. 6a reports the result for the piezoresistive device, showing no visible effects from vibrations. For comparison, Fig. $6 \mathrm{~b}$ reports the same result for the off-the-shelf capacitive gyroscope: the result clearly highlights poor vibration rejection (spikes as large as $250 \mathrm{dps}$ ) at several frequencies. Note the consistently different vertical scale.
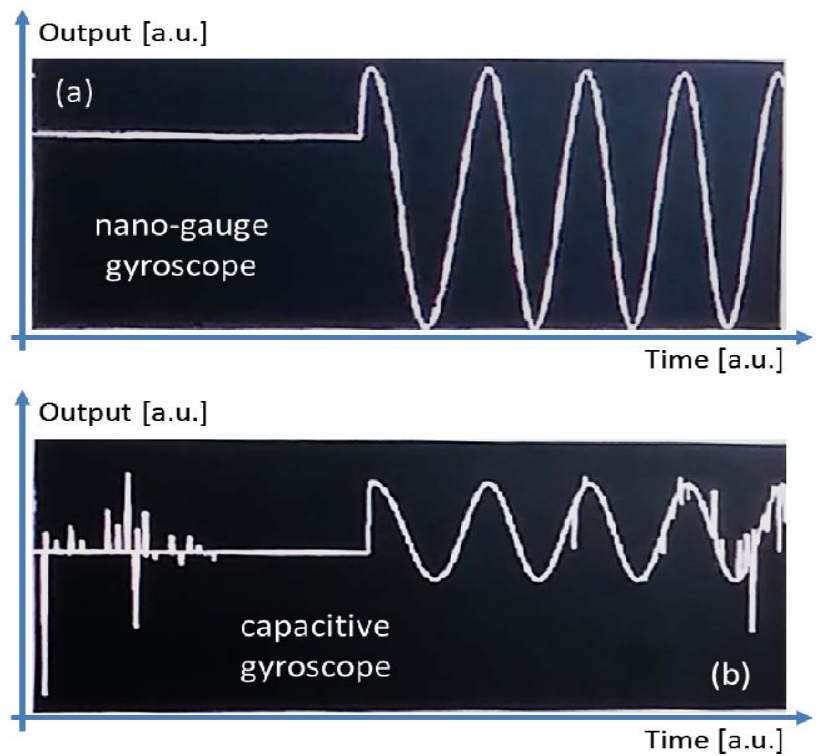

Fig. 7: output acquired during a sinusoidal angular rate with superimposed vibrations for (a) the proposed piezoresistive gyroscopes and (b) the reference gyroscope. 
Fig. 7 reports the results of the application of strong mechanical shocks during the acquisition of sinusoidal rates with an amplitude of $100 \mathrm{dps}$. Again, the performance of the piezoresistive device results consistently better.

To quantify the observed differences in terms of vibration rejection, Allan variance measurements were performed on both devices. The Allan variance method is commonly used to describe the noise and long-term stability of MEMS gyroscopes [5, 7-10]. It indeed gives a synthetic representation of the different noise contributions of the system, that become visible as a function of the observation time intervals. Computing the Allan variance at different time intervals means band-pass filtering the considered signal at different passing frequencies. In this way both fast (e.g. white noise) and slow contributions (e.g. temperatureinduced or other kinds of drift) are well distinguishable. Its extended usage in literature is due to its simplicity of implementation and effectiveness of interpretation.

It is worth noting, however, that data measured in a laboratory environment (even if uncontrolled) may be not representative of real operating conditions, where e.g. temperature drifts or vibrations may appear together with accelerations and rotations. In particular, only a few works $[1,2]$ discussed the relevance of vibration immunity for MEMS gyroscopes.

For the specific situation of this work, Fig. 8 reports the consequences of the application of vibrations on the Allan variance graphs, showing the measurement in presence and in absence of the 1-10 kHz vibratory sweep of Fig. 6.

For the capacitive device, the $50 \mathrm{mdps} / \sqrt{\mathrm{Hz}}$ ARW increases by two orders of magnitude, likely due the presence of modes in the range $<10 \mathrm{kHz}$. On the contrary, for the piezoresistive gyroscope the $4.5 \mathrm{mdps} / \sqrt{\mathrm{Hz}} \mathrm{ARW}$ shows no noticeable change. A worst-case $5 \mathrm{x}$ bias stability increase is seen at observation times of a few s. The measurement confirms a high vibration rejection for the proposed piezoresistive gyroscope. Future work includes the extension of this experiment to the $50 \mathrm{kHz}$ range.

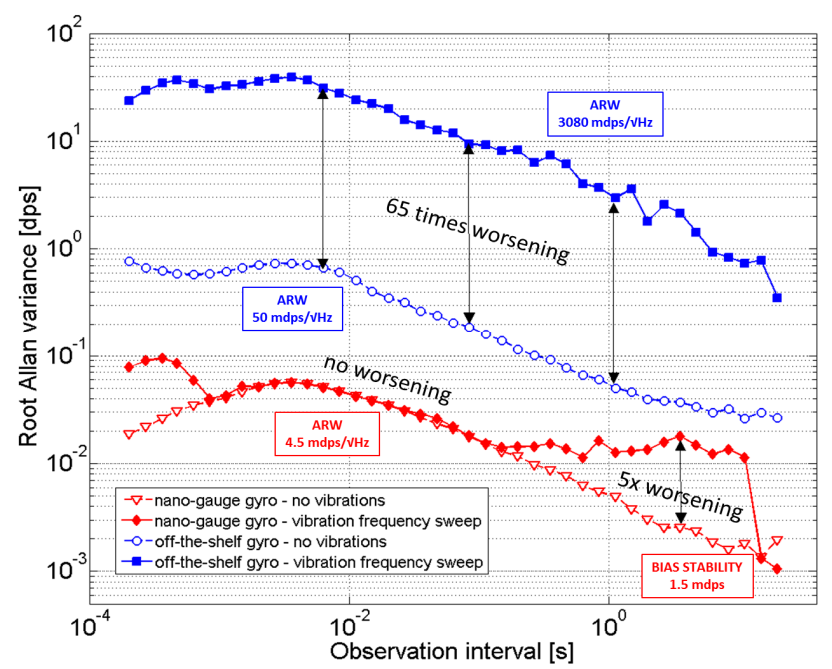

Fig. 8: Allan variance graphs acquired with and without applying the vibrations of Fig. 6, for the two gyroscope types described in this work.

\section{ACKNOWLEDGEMENTS}

The work is supported by the European Union under grant 288318, "Nine-axis inertial sensors based on piezoresistive nanogauge Detection". Experiments were done in the DEIB facilities of the MEMS\&3D lab. The authors acknowledge P. Robert from CEA-Leti for helping with the equipment.

\section{REFERENCES}

[1] H Weinberg, "Gyro Mechanical Performance: The Most Important Parameter," Analog Device technical article MS-2158, Sept. 2011.

[2] S. W. Yoon, S. Lee, K. Najafi, "Vibration-induced errors in MEMS tuning fork gyroscopes," Sensors and Actuators A 180 (2012), pp. 32-44.

[3] C. Acar, A. R. Schofield, A. A. Trusov, L. E. Costlow, A. M. Shkel, "Environmentally Robust MEMS Vibratory Gyroscopes for Automotive Applications," IEEE Sensors Journal, vol.9, n.12, pp.1895-1906, Dec.2009.

[4] N. El-Sheimy, H. Haiying, N. Xiaoji, "Analysis and Modeling of Inertial Sensors Using Allan Variance," IEEE Tran. Instrumentation and Measurement, vol. 57, n.1, pp.140-149, Jan 2008.

[5] S. Dellea, F. Giacci, A. Longoni, P. Rey, A. Berthelot, G. Langfelder, "Large full scale, linearity and cross-axis rejection in low-power 3axis gyroscopes based on nanoscale piezoresistors," proc. MEMS 2015, pp. 37-40, Lisbon, Portugal, Jan. 2015.

[6] P. Robert, V. Nguyen, S. Duraffourg, G. Jourdan, J. Arcamone, S. Harrison, "M\&NEMS : A new approach for ultra-low cost $3 D$ inertial sensor," proc. IEEE Sensors 2009, pp. 963-966, Christchurch, New Zealand, Oct. 2009.

[7] V. Kempe, "Gyroscopes, Chapter 8 in Inertial MEMS Principles and Practice," Cambridge University press, 2011.

[8] J. A. Geen, S. Sherman, J. F. Chang, S. R. Lewis, "Single-Chip Surface Micromachined Integrated Gyroscope With 50\% Allan Deviation," IEEE Journ. Solid-State Circ., vol. 37, n. 12, Dec 2002, pp. 1860-1866.

[9] A. Walther, M. Savoye, et al., "3-axis Gyroscope with Si nanogage piezo-resistive detection," proc. MEMS 2012, p. 480-483, Paris, France, Jan 2012.

[10] M. Vagner, P. Benes, Z. Havranek, "Experience With Allan Variance Method for MEMS Gyroscope Performance Characterization," IEEE I2MTC 2012 Conference, pp.1343-1347, 2012.

\section{CONTACT}

F. Giacci, tel: +39-02-23993425; federico.giacci@polimi.it. 